\title{
Spectra of weighted composition operators on abstract Hardy spaces
}

\author{
Ted Eklund ${ }^{1}$ - Mikael Lindström ${ }^{1}$. \\ Paweł Mleczko $^{2}$ (D) Michał Rzeczkowski ${ }^{2}$
}

Received: 14 July 2017 / Accepted: 13 November 2017 / Published online: 21 November 2017

(C) The Author(s) 2017. This article is an open access publication

\begin{abstract}
In the paper, the spectra of weighted composition operators on Hardy-type spaces of holomorphic functions on the unit disc of the complex plane are studied. The spectra of invertible operators induced by elliptic and parabolic automorphisms are described, for weighted composition operators acting on abstract Hardy spaces generated by Banach lattices. We also study spectra of weighted composition operators (not necessarily invertible) on Hardy-Lorentz spaces.
\end{abstract}

Keywords Weighted composition operator - Spectrum · Automorphism - Hardy spaces · Rearrangement invariant spaces

Mathematics Subject Classification Primary 47B33; Secondary 47B35

\section{Introduction}

Let $H(\mathbb{D})$ denote the space of holomorphic functions on the unit disc $\mathbb{D}$ of the complex plane $\mathbb{C}$. Any functions $u, \varphi \in H(\mathbb{D}), \varphi(\mathbb{D}) \subset \mathbb{D}$, induce a mapping $u C_{\varphi}: H(\mathbb{D}) \rightarrow H(\mathbb{D})$ called a weighted composition operator, where

Paweł Mleczko

pml@amu.edu.pl

Ted Eklund

ted.eklund@abo.fi

Mikael Lindström

mikael.lindstrom@abo.fi

Michał Rzeczkowski

rzeczkow@amu.edu.pl

1 Department of Mathematics, Åbo Akademi University, 20500 Åbo, Finland

2 Faculty of Mathematics and Computer Science, Adam Mickiewicz University in Poznań, Umultowska 87, 61-614 Poznan, Poland 


$$
u C_{\varphi} f(z)=u(z) f(\varphi(z)), \quad f \in H(\mathbb{D}), z \in \mathbb{D} .
$$

If $u \equiv 1$, then the corresponding map $C_{\varphi}$ is called a composition operator.

The study of composition operators on various spaces of holomorphic functions was initiated at the beginning of the twentieth century by the works of Hardy, Littlewood, and Riesz (see [6]). Over the years the questions related to the operator-theoretic properties of $u C_{\varphi}$ have been a great motivation for the development of complex and functional analysis. Very recently the spectra of weighted composition operators on different spaces of holomorphic functions have been extensively studied. Most research was done with $\varphi$ an automorphism of $\mathbb{D}$, and the main goal was to give a description of the spectrum of $u C_{\varphi}$ in terms of the functions $\varphi$ and $u$. In this respect the main achievement was obtained for the Hardy, Bergman, and growth spaces (see [11]). The same problem has recently been considered for other types of spaces of holomorphic functions, namely the Dirichlet (see [5,9]) and the Bloch spaces (see [9]).

Moreover, weighted composition operators (and their operator-theoretic properties) have recently been treated in the context of more abstract Hardy classes, like Hardy-Orlicz spaces (see for example [15]), and general Hardy spaces generated by rearrangement-invariant Banach spaces (see [19]).

The aim of this paper is to study the spectra of weighted composition operators on abstract variants of Hardy spaces. The paper is organized as follows. In the preliminary section we gather the essential notions and discuss in detail the tools we will use underneath. The main results of this paper are contained in Sect. 3, where the study of the spectra of weighted composition operators $u C_{\varphi}$ on abstract Hardy spaces is presented, when the generating symbol $\varphi$ is an automorphism of the disc. A complete description of the spectra for Hardy-Lorentz spaces is given, and then a characterization of spectra of weighted composition operators $u C_{\varphi}$ on abstract Hardy spaces is provided with $\varphi$ parabolic or elliptic. In the final section, it is shown that the spectra of weighted composition operators on Hardy-Lorentz and Hardy spaces coincide.

\section{Preliminaries}

Let $(\Omega, \Sigma, \mu)$ be a complete $\sigma$-finite measure space and let $L^{0}(\Omega):=L^{0}(\Omega, \Sigma, \mu)$ denotes the space of real valued measurable functions on $\Omega$ with the topology of convergence in measure on $\mu$-finite sets. The order $|f| \leqslant|g|$ means that $|f(\omega)| \leqslant|g(\omega)|$ for $\mu$-almost all $\omega \in \Omega$. If a real Banach space $X \subset L^{0}(\Omega)$ is such that there exists $f \in X$ with $f>0$ $\mu$-a.e. on $\Omega$ and $|f| \leqslant|g|$ with $f \in L^{0}(\Omega)$ and $g \in X$ implies $f \in X$ with $\|f\|_{X} \leqslant\|g\|_{X}$, then $X$ is said to be a Banach lattice (on $\Omega$ or on $(\Omega, \mu)$ ). An important class of Banach lattices are rearrangement-invariant spaces. Given $f \in L^{0}(\Omega)$, its distribution function is given by $\mu_{f}(\lambda)=\mu(\{t \in \Omega:|f(t)|>\lambda\}), \lambda \geqslant 0$. A Banach lattice $X$ is called to be rearrangement invariant (r.i. space for short) if for any $f \in X$ and $g \in L^{0}(\Omega)$ such that $\mu_{f}=\mu_{g}$ we have $g \in X$ and $\|f\|_{X}=\|g\|_{X}$. It is well known that if $X$ is an r.i. space for some finite measure space $\Omega$, then (see [14])

$$
L^{\infty}(\Omega) \hookrightarrow X \hookrightarrow L^{1}(\Omega) .
$$

Throughout the paper we will consider complex r.i. spaces. The term complex ri. space refers to the complexification of a real r.i. space, i.e., if $X$ denotes the (real) r.i. space, the complexification $X(\mathbb{C})$ of $X$ is the Banach space of all complex valued measurable functions $f$ on $\Omega$ such that the element $|f|$ defined by $|f|(\omega)=|f(\omega)|$ for $\omega \in \Omega$ is in $X$ and 
$\|f\|=\||f|\|_{X}$. For simplicity of presentation, we will often write ri. space instead of complex r.i. space and avoid using the symbol $X(\mathbb{C})$. An r.i. space $X$ is said to be maximal (or to have the Fatou property), if for any sequence $\left(f_{n}\right)$ of non-negative elements from $X$ such that $f_{n} \uparrow f$ for $f \in L^{0}(\Omega)$ and $\sup \left\{\left\|f_{n}\right\|_{X}: n \in \mathbb{N}\right\}<\infty$, one has $f \in X$ and $\left\|f_{n}\right\|_{X} \rightarrow\|f\|_{X}$.

If $X$ is an r.i. space then for any measurable set $B$, the expression $\left\|\chi_{B}\right\|$ depends only of $\mu(B)$. Thus for every $t \in\{\mu(B): B \in \Sigma\}$ we define a function $\phi_{X}$ by the formula $\phi_{X}(t)=\left\|\chi_{[0, t]}\right\|$. This function is called the fundamental function of $X$.

We collect here the basic properties of $\phi_{X}$. If $X$ is an r.i. function space on a non-atomic measure space $(\Omega, \Sigma, \mu)$, then $\phi_{X}$ is quasi-concave on $[0, \tau)$ with $\tau=\mu(\Omega)$, i.e., $\phi_{X}(0)=0$, $\phi_{X}$ is positive, non-decreasing, and $t \mapsto \phi_{X}(t) / t$ is non-increasing on $(0, \tau)$ (see [14]). Notice that for a quasi-concave function $\psi$ there exists a concave function $\bar{\psi}$ given by $\bar{\psi}(t)=\inf \left\{\left(1+\frac{1}{s}\right) \psi(s): s \in(0, \tau)\right\}$ such that $\psi(t) \leqslant \bar{\psi}(t) \leqslant 2 \psi(t)$ for $t \in[0, \tau)$. Thus we may assume that $\phi_{X}$ is a concave function. Note also that $\phi_{X}$ is continuous at 0 if and only if $X \neq L^{\infty}$.

\subsection{Interpolation of operators}

In the Sect. 4 we will use interpolation theory to study the spectra of weighted composition operators. Here we present some basic concepts; for a more detailed study we refer the reader to the monograph [3].

Let $E_{0}$ and $E_{1}$ be Banach spaces. The pair $\vec{E}=\left(E_{0}, E_{1}\right)$ is called a Banach couple if there exists a Hausdorff topological vector space $\mathcal{X}$ such that $E_{j} \hookrightarrow \mathcal{X}, j=0,1$. A Banach space $E$ is called intermediate space with respect to $\vec{E}$, if $E_{0} \cap E_{1} \hookrightarrow E \hookrightarrow E_{0}+E_{1}$. Let $\vec{E}=\left(E_{0}, E_{1}\right)$ and $\vec{F}=\left(F_{0}, F_{1}\right)$ be Banach couples. If for any intermediate spaces $E$ and $F$ with respect to $\vec{E}$ and $\vec{F}$, and every linear map $T: E_{0}+E_{1} \rightarrow E_{0}+E_{1}$ such that $\left.T\right|_{E_{j}}$ is a bounded operator from $E_{j}$ into $F_{j}, j=0,1$, the operator $T: E \rightarrow F$ is bounded, then $E$ and $F$ are called interpolation spaces (with respect to $\vec{E}$ and $\vec{F}$ ).

For a Banach couple $\vec{E}$ define a $K$-functional by the formula

$$
K(t, x ; \vec{E})=\inf \left\{\left\|x_{0}\right\|_{E_{0}}+t\left\|x_{1}\right\|_{E_{1}}: x=x_{0}+x_{1}, x_{0} \in E_{0}, x_{1} \in E_{1}\right\},
$$

where $t>0, x \in E_{0}+E_{1}$. For $\theta \in(0,1)$ and $q \in[1, \infty]$ the real interpolation space $\left(E_{0}, E_{1}\right)_{\theta, q}$ consists of those $x \in E_{0}+E_{1}$ for which

$$
\|x\|_{\theta, q}= \begin{cases}\left(\int_{0}^{\infty}\left(\frac{1}{t^{\theta}} K(t, x ; \vec{E})\right)^{q} \frac{d t}{t}\right)^{1 / q}, & q \in[1, \infty) \\ \sup _{t>0} \frac{1}{t^{\theta}} K(t, x ; \vec{E}), & q=\infty .\end{cases}
$$

In applications, r.i. Lorentz spaces appear as a natural object of study (see [10]). Given a non-atomic measure space $(\Omega, \Sigma, \mu)$ and $p \in(1, \infty), q \in[1, \infty]$, the Lorentz space $L^{p, q}$ on $(\Omega, \Sigma, \mu)$ consists of all $f \in L^{0}(\Omega)$ such that

$$
\|f\|_{L^{p, q}}= \begin{cases}\left(\int_{0}^{\mu(\Omega)}\left(t^{1 / p} f^{* *}(t)\right)^{q} \frac{d t}{t}\right)^{1 / q}, & q<\infty \\ \sup _{t \in(0, \mu(\Omega))} t^{1 / p} f^{* *}(t), & q=\infty\end{cases}
$$

where $f^{* *}(t)=\frac{1}{t} \int_{0}^{t} f^{*}(s) d s, t>0$ and $f^{*}$ denotes the non-increasing rearrangement of $f$ (i.e., $f^{*}(t)=\inf \left\{\lambda>0: \mu_{f}(\lambda) \leqslant t\right\}, t \geqslant 0$ ). 


\subsection{Hardy spaces on a disc}

Let $X$ be an r.i. space on $\mathbb{T}:=[0,2 \pi)$ with normalized Lebesgue measure. An abstract Hardy space $H X=H X(\mathbb{D})$ consists of holomorphic functions on the unit disc $\mathbb{D}$ such that

$$
\sup _{r \in[0,1)}\left\|f_{r}\right\|_{X}<\infty
$$

where $f_{r}(t):=f\left(r e^{i t}\right)$ for $t \in \mathbb{T}$ and $r \in[0,1)$. With the norm $\|\cdot\|_{H X}$ given by $\|f\|_{H X}:=$ $\sup \left\{\left\|f_{r}\right\|_{X}: r \in[0,1)\right\}, H X$ is a Banach space. For a study of general variants of Hardy spaces, see $[18,19]$. For particular spaces $X$, the above method produces variants of Hardy spaces widely studied in the literature. For example, if $X=L^{p}, p \in[1, \infty]$, then $H X$ is the standard Hardy space $H^{p}$ (see [6]).

We shall give two important examples of r.i. spaces, namely Lorentz and Orlicz spaces. Let $\psi$ be a positive, increasing and concave function on $[0, \tau)$. Let $(\Omega, \Sigma, \mu)$ be a non-atomic measure space. The Lorentz space $\Lambda(\psi)$ consists of all $f \in L^{0}(\mu)$ such that

$$
\|f\|_{\Lambda(\psi)}=\int_{0}^{\tau} f^{*}(t) d \psi(t)<\infty,
$$

It is clear that the fundamental function of the Lorentz space $\Lambda(\psi)$ equals $\psi$.

Let $\Phi$ be an Orlicz function, i.e., $\Phi:[0, \infty) \rightarrow[0, \infty)$, continuous and convex. Then the Orlicz space $L^{\Phi}$ consists of the elements from $L^{0}(\mu)$ such that

$$
\|f\|_{L^{\Phi}}:=\inf \left\{\varepsilon>0: \int_{\Omega} \Phi\left(\frac{|f|}{\varepsilon}\right) d \mu \leqslant 1\right\}<\infty .
$$

Note that in the case $\Omega=\mathbb{T}$, the Hardy-Lorentz space $H^{\psi}$ is defined as $H^{\psi}:=H \Lambda(\psi)$ (see [16]). If $p \in[1, \infty)$ and $\psi(t)=t^{1 / p}$ for all $t \in[0,1]$, then we obtain the classical Lorentz spaces $L^{p, 1}$. The Hardy-Orlicz spaces $H^{\Phi}$ are given by $H^{\Phi}:=H L^{\Phi}$ (see [15]).

The systematic study of composition operators on spaces more general than the classical Hardy spaces $H^{p}$ dates back to the papers on Hardy-Orlicz spaces by Lefèvre, Li, Queffélec, and Rodríguez-Piazza from the beginning of the twenty-first century (see the book [15] and the list of references therein). Recently, Carleson embeddings of abstract Hardy spaces into Banach lattices with an application to the study of analytic properties of composition operators on very general Hardy classes have been treated by Mastyło and Rodríguez-Piazza in [19]. In the latter paper it was proved, among other things, that if $X$ is a maximal r.i. space on $\mathbb{T}$ then every $\varphi \in H(\mathbb{D}), \varphi(\mathbb{D}) \subset \mathbb{D}$, induces a composition operator $C_{\varphi}: H X \rightarrow H X$ (see [19, Proposition 1.4]) and

$$
\left\|C_{\varphi}\right\| \leqslant \frac{1+|\varphi(0)|}{1-|\varphi(0)|} .
$$

In what follows, we will need a particular estimate of the norm of the evaluation functional $\delta_{z}$ on $H X$. Notice that if $X$ is an r.i. space and $\phi_{X}$ is a fundamental function, then the following inequalities, proved in [19, Lemma 1.2], hold:

$$
\frac{1}{4 \phi_{X}(1-|z|)} \leqslant\left\|\delta_{z}\right\|_{(H X)^{*}} \leqslant \frac{2}{\phi_{X}(1-|z|)}, \quad z \in \mathbb{D} .
$$

Note that from these inequalities follow the well-known estimates of the evaluation functionals since the fundamental functions can be written explicitly. Indeed, if $X=L^{p}(\mathbb{T})$, then $\phi_{L^{p}}(t)=t^{1 / p}$ for $t \in[0,1]$, while for an Orlicz function $M, \phi_{L^{M}}(t)=\left(M^{-1}(1 / t)\right)^{-1}$ for $t \in[0,1]$. 


\subsection{Motivation}

In $[11,12]$, the spectra of composition operators were studied within the framework of a very general axiomatic approach. One of the key assumptions was that the space $E \subset H(\mathbb{D})$ satisfy the following condition:

(A) There is a positive constant $s$ (depending on $E$ ) such that for $f \in E$ and $z \in \mathbb{D}$ we have $|f(z)| \leqslant\|f\|\left(1-|z|^{2}\right)^{-s}$.

It appears that there are important spaces for which the above condition does not hold. For example, this is the case for a Hardy-Orlicz space. Indeed, it follows from (2) that for the Hardy-Orlicz space $H^{M}$, the following estimate is true $|f(z)| \leqslant 2 M^{-1}(1 /(1-|z|))$. The main goal of this paper is to study the spectra of weighted composition operators on Hardy spaces generated by r.i. spaces on $\mathbb{T}$. In other words, (cf. (2)) we will study spaces of analytic functions satisfying the property:

$\left(\mathrm{A}^{\prime}\right)$ There exists a concave function $\phi_{X}$ (depending on $X$ ) such that for every $f \in X$

$$
|f(z)| \leqslant C \frac{\|f\|}{\phi_{X}(1-|z|)}, \quad z \in \mathbb{D} \text {. }
$$

Our proofs rely on the approach taken in [9] (cf. also [11]). Let us note, however, that the much more complicated structure of the norm of the spaces under consideration forced us to take a slightly different approach. Results from Sect. 4 are obtained utilizing the outcome of $[1,2]$.

\subsection{Multiplication operator on $H X$}

In what follows we will need a description of a bounded multiplication operator. The following result is well known for the classical Hardy spaces $H^{p}$ and, for example, for Hardy-Orlicz spaces (see [17]). Below we enclose the proof of the general variant.

Theorem 1 Let $X$ be a maximal ri. space on $\mathbb{T}$ and suppose that $u \in H(\mathbb{D})$. Then the following statements are equivalent:

(i) $u H X \subset H X$

(ii) $u \in H X$ and the operator $M_{u}$ is bounded

(iii) $u \in H^{\infty}$.

Moreover, $\left\|M_{u}: H X \rightarrow H X\right\|=\|u\|_{\infty}$.

Proof (i) $\Rightarrow$ (ii). From the estimates (2) it follows that the convergence in $H X$ norm is stronger than the uniform convergence on compact sets in $\mathbb{D}$. Hence $M_{u}$ has a closed graph and it follows from the Closed Graph Theorem that $M_{u}: H X \rightarrow H X$ is bounded.

(ii) $\Rightarrow$ (iii). Choose $z \in \mathbb{D}$ and let us consider the functional $\delta_{z}$ given for $f \in H X$ by $\delta_{z} f=f(z)$. It is clear that $\delta_{z}\left(M_{u}(f)\right)=u(z) f(z)$ and it follows from (2) that $\delta_{z} \in(H X)^{*}$. Since $\left(M_{u}^{*} \delta_{z}\right) f=u(z) f(z)=\left(u(z) \delta_{z}\right) f$, then $\left(M_{u}^{*} \delta_{z}-u(z) \delta_{z}\right)(f)=0$. We have just noticed that $M_{u}^{*} \delta_{z}=u(z) \delta_{z}$. To conclude, observe that $\left\|M_{u}^{*} \delta_{z}\right\|_{(H X)^{*}}=|u(z)|\left\|\delta_{z}\right\|_{(H X)^{*}} \leqslant$ $\left\|M_{u}^{*}\right\|\left\|\delta_{z}\right\|_{(H X)^{*}}$. This yields

$$
|u(z)| \leqslant\left\|M_{u}: H X \rightarrow H X\right\|,
$$

as required. 


\section{Spectra of invertible weighted composition operators on abstract Hardy spaces}

In this section we study the spectra of invertible weighted composition operators induced by parabolic or elliptic symbols. The next theorem, which follows directly from Bourdon's general result (see [4, Corollary 2.3], characterizes operators of this type.

Theorem 2 Let $X$ be an ri. space on $\mathbb{T}$ and suppose that $u C_{\varphi}: H X \rightarrow H X$ is a weighted composition operator. Then $u C_{\varphi}$ is invertible if and only if $u \in H^{\infty}, u$ is bounded away from zero on $\mathbb{D}$, and $\varphi$ is an automorphism of $\mathbb{D}$. Moreover, if this is the case, then the inverse is also a weighted composition operator and

$$
\left(u C_{\varphi}\right)^{-1}=\frac{1}{u \circ \varphi^{-1}} C_{\varphi^{-1}} .
$$

The spectral analysis of invertible weighted composition operators $u C_{\varphi}$ depends significantly on the dynamics of the symbol $\varphi$ (see for example $[9,11]$ ). Recall that the automorphism of $\mathbb{D}$ is called (see [6])

- elliptic if $\varphi$ has a unique fixed point in $\mathbb{D}$,

- parabolic if $\varphi$ has a unique fixed point in $\partial \mathbb{D}$,

- hyperbolic if $\varphi$ has two fixed points in $\partial \mathbb{D}$.

Recall that the famous Denjoy-Wolff Theorem (see [6, Theorem 2.51]) asserts that when $\varphi$ is a parabolic or hyperbolic automorphism of $\mathbb{D}$, then there is a unique fixed point $a \in \partial \mathbb{D}$ such that

$$
\lim _{n \rightarrow \infty} \varphi_{n}(z)=a
$$

uniformly on compact subsets of $\mathbb{D}$, where

$$
\varphi_{n}:=\varphi \circ \varphi_{n-1}, \quad n \geqslant 2,
$$

and $\varphi_{1}:=\varphi$. The point $a$ is called the Denjoy-Wolff point (or attractive fixed point) of $\varphi$. Furthermore when $\varphi$ is parabolic, then $\varphi^{\prime}(a)=1$. When $\varphi$ is hyperbolic and its other fixed point is $b \in \partial \mathbb{D}$ (called repulsive fixed point), then $0<\varphi^{\prime}(a)<1$ and $\varphi^{\prime}(b)=1 / \varphi^{\prime}(a)$. Moreover (see [6, pp. 251-252])

$$
\lim _{n \rightarrow \infty}\left(1-\left|\varphi_{n}(0)\right|\right)^{1 / n}=\varphi^{\prime}(a),
$$

where $\varphi$ is a parabolic or hyperbolic automorphism of the unit disk. For any $n \geqslant 1$ we have

$$
\left(u C_{\varphi}\right)^{n} f(z)=u(z) \cdots u\left(\varphi_{n-1}(z)\right) f\left(\varphi_{n}(z)\right), \quad f \in H(\mathbb{D}), \quad z \in \mathbb{D} .
$$

From this it follows that $\left(u C_{\varphi}\right)^{n}=u_{(n)} C_{\varphi_{n}}$, where

$$
u_{(n)}=\prod_{m=0}^{n-1} u \circ \varphi_{m}, \quad n \geqslant 1,
$$

and $u_{(0)}=1$. Throughout the paper the symbol $A(\mathbb{D})$ denotes the disc algebra. 


\subsection{The parabolic case}

In this section we will give a description of the spectrum of weighted composition operator $u C_{\varphi}: H X \rightarrow H X$, where $X$ is an r.i. space and $\phi: \mathbb{D} \rightarrow \mathbb{D}$ is a parabolic automorphism. The special case when $X=L^{p}, p \in[1, \infty)$ was treated in [11, Section 4.1].

In the proof of the main theorem of this section we will use the following lemma.

Lemma 1 Let $\psi:[0, \tau) \rightarrow[0, \tau)$ with $\tau>0$ be a quasi-concave function. Let $\left\{a_{n}\right\},\left\{b_{n}\right\} \subset$ $(0, \tau)$ be sequences such that $a_{n} \rightarrow 0, b_{n} \rightarrow 0$, as $n \rightarrow \infty$. If

$$
\lim _{n \rightarrow \infty} \frac{a_{n}}{b_{n}}=1
$$

then

$$
\lim _{n \rightarrow \infty} \frac{\psi\left(a_{n}\right)}{\psi\left(b_{n}\right)}=1
$$

Proof Since $a_{n} / b_{n} \rightarrow 1$ and $b_{n} / a_{n} \rightarrow 1$ as $n \rightarrow \infty$, it follows that for every $\varepsilon \in(0,1)$ there exists $n_{0} \in \mathbb{N}$ such that for each $n>n_{0}$ we have $a_{n} \leqslant(1+\varepsilon) b_{n}$ and $b_{n} \leqslant(1+\varepsilon) a_{n}$. For a given $n>n_{0}$ we have either $a_{n} \geqslant b_{n}$ or $a_{n}<b_{n}$. In the first case we obtain by quasi-concavity of $\psi$

$$
\left|\psi\left(a_{n}\right)-\psi\left(b_{n}\right)\right| \leqslant \psi\left((1+\varepsilon) b_{n}\right)-\psi\left(b_{n}\right) \leqslant(1+\varepsilon) \psi\left(b_{n}\right)-\psi\left(b_{n}\right)=\varepsilon \psi\left(b_{n}\right)
$$

and so

$$
1-\varepsilon<\frac{\psi\left(a_{n}\right)}{\psi\left(b_{n}\right)} \leqslant 1+\varepsilon
$$

In the second case (that is, when $a_{n}<b_{n}$ ) we obtain

$$
\frac{\psi\left(b_{n}\right)}{\psi\left(a_{n}\right)} \leqslant 1+\varepsilon
$$

Since in this case $\psi\left(a_{n}\right) \leqslant \psi\left(b_{n}\right)$,

$$
1-\varepsilon<\frac{1}{1+\varepsilon} \leqslant \frac{\psi\left(a_{n}\right)}{\psi\left(b_{n}\right)} \leqslant 1<1+\varepsilon .
$$

As a result for a given $\varepsilon \in(0,1)$ there exists $n_{0}$ such that

$$
\left|\frac{\psi\left(a_{n}\right)}{\psi\left(b_{n}\right)}-1\right|<\varepsilon, \quad n>n_{0}
$$

and so

$$
\lim _{n \rightarrow \infty} \frac{\psi\left(a_{n}\right)}{\psi\left(b_{n}\right)}=1
$$

Recall that an interpolating sequence for $H^{\infty}(\mathbb{D})$ is a sequence $\left\{z_{j}\right\} \subset \mathbb{D}$ such that for any bounded sequence of complex numbers $\left\{c_{j}\right\}$, there is a function $f \in H^{\infty}(\mathbb{D})$ such that $f\left(z_{j}\right)=c_{j}$. It was proved in [7, Proposition 4.9] that if $z_{0} \in \mathbb{D}$, then the sequence of iterates $\left\{\varphi_{n}\left(z_{0}\right)\right\}, n \in \mathbb{N}$, is an interpolating sequence for $H^{\infty}(\mathbb{D})$. 
Theorem 3 Let $u C_{\varphi}: H X \rightarrow H X$ be an invertible weighted composition operator and assume that $\varphi$ is a parabolic automorphism of the disc $\mathbb{D}$ with a unique fixed point $a \in \partial \mathbb{D}$. If $u \in A(\mathbb{D})$, then

$$
r_{H X}\left(u C_{\varphi}\right)=|u(a)|
$$

and

$$
\sigma_{H X}\left(u C_{\varphi}\right)=\{z \in \mathbb{C}:|z|=|u(a)|\} .
$$

Proof To prove that $r\left(u C_{\varphi}\right) \leqslant|u(a)|$ we note that by (1) it follows that

$$
\left\|C_{\varphi_{n}}\right\| \leqslant \frac{1+\left|\varphi_{n}(0)\right|}{1-\left|\varphi_{n}(0)\right|} \leqslant \frac{2}{1-\left|\varphi_{n}(0)\right|} .
$$

Since $\varphi$ is a parabolic automorphism by (3) we conclude that

$$
\left\|C_{\varphi_{n}}\right\|^{\frac{1}{n}} \leqslant\left(\frac{2}{1-\left|\varphi_{n}(0)\right|}\right)^{\frac{1}{n}} \rightarrow \frac{1}{\varphi^{\prime}(a)}=1 \text { as } n \rightarrow \infty .
$$

Now from Theorem 1 it follows that

$$
\left\|\left(u C_{\varphi}\right)^{n}\right\|=\left\|M_{u_{(n)}} C_{\varphi_{n}}\right\| \leqslant\left\|M_{u_{(n)}}\right\|\left\|C_{\varphi_{n}}\right\|=\left\|u_{(n)}\right\|_{\infty}\left\|C_{\varphi_{n}}\right\| .
$$

Combining the above considerations with the formula $\left\|u_{(n)}\right\|_{\infty}^{\frac{1}{n}} \rightarrow|u(a)|$ as $n \rightarrow \infty$ (see [11, Lemma 4.2]), we conclude that

$$
r\left(u C_{\varphi}\right) \leqslant|u(a)| .
$$

We will prove the reverse inequality. Let $a \in \partial \mathbb{D}$ be the fixed point of $\varphi$ and take $\varepsilon \in(0,1)$. Notice that (see [11, p. 1759])

$$
\left\|\left(u C_{\varphi}\right)^{n}\right\|=\left\|u_{(n)} C_{\varphi_{n}}\right\| \geqslant \frac{\left|u_{(n)}(\varepsilon a)\right|\left\|\delta_{\varphi_{n}(\varepsilon a)}\right\|_{(H X)^{*}}}{\left\|\delta_{a}\right\|_{(H X)^{*}}}
$$

From the formula (2) it follows that for every $\varepsilon \in(0,1)$ we have

$$
\begin{aligned}
\left\|\left(u C_{\varphi}\right)^{n}\right\| & \geqslant\left|u_{(n)}(\varepsilon a)\right| \frac{\frac{1}{4 \phi_{X}\left(1-\left|\varphi_{n}(\varepsilon a)\right|\right)}}{\frac{2}{\phi_{X}(1-|\varepsilon a|)}} \\
& \geqslant\left|u_{(n)}(\varepsilon a)\right| \frac{\phi_{X}(1-|\varepsilon a|)}{2 \phi_{X}\left(1-\left|\varphi_{n}(\varepsilon a)\right|\right)} .
\end{aligned}
$$

Since $\phi_{X}$ is a quasi-concave function on $[0,1)$, the inequality $r\left(u C_{\varphi}\right) \geqslant|u(a)|$ now follows from Lemma 1 (with $\tau=1$ ) and the fact that

$$
\lim _{\varepsilon \rightarrow 1^{-}}\left|u_{(n)}(\varepsilon a)\right|=|u(a)|^{n} .
$$

To give a description of the spectrum of $u C_{\varphi}: H X \rightarrow H X$, observe that it is enough to justify the inclusion

$$
\left\{\lambda:|\lambda| \in r\left(u C_{\varphi}\right)\right\} \subset \sigma_{H X}\left(u C_{\varphi}\right) .
$$

Indeed (cf. [11, p. 1761]), observe that $\left(u C_{\varphi}\right)^{-1}=\frac{1}{u \circ \varphi^{-1}} C_{\varphi^{-1}}$ and $\varphi^{-1}$ is also a parabolic automorphism with the same fixed point $a \in \mathbb{D}$. Now take $\lambda \in \sigma_{H X}\left(u C_{\varphi}\right)$. Note that $\lambda^{-1} \in$ 
$\sigma_{H X}\left(\frac{1}{u \circ \varphi^{-1}} C_{\varphi^{-1}}\right)$ and conclude that $|\lambda|^{-1} \leqslant|u(a)|^{-1}$. Hence $|u(a)| \leqslant|\lambda|$ and by the inequality $|\lambda| \leqslant r\left(u C_{\varphi}\right)=|u(a)|$ it follows that

$$
\sigma_{H X}\left(u C_{\varphi}\right) \subset\left\{\lambda:|\lambda|=r\left(u C_{\varphi}\right)\right\} .
$$

We will prove the formula (8). Take a sequence $\left\{z_{n}\right\}$ defined by $z_{n}=\left\{\varphi_{n}(0)\right\}, n \in \mathbb{N}_{0}$ (cf. [11, Section 4.1]). Since $\left\{z_{n}\right\}$ is an interpolating sequence for $H^{\infty}$, by the Open Mapping Theorem there exist a constant $c>0$ and a sequence $\left\{f_{n}\right\} \subset H^{\infty}$ such that $\left\|f_{n}\right\|_{\infty} \leqslant c$ for all $n \in \mathbb{N}_{0}$ and

$$
f_{n}\left(\varphi_{k}\left(z_{n}\right)\right)= \begin{cases}1, & \text { if } k=n \\ 0, & \text { if } k \neq n .\end{cases}
$$

Since $L^{\infty} \hookrightarrow X \hookrightarrow L^{1}$, it follows that the sequence $\left\{f_{n}\right\}$ is bounded in $H X$ and hence also in $H^{1}$. Take $\lambda \in \mathbb{C}$ with $|\lambda|=|u(a)|$. From the above we get

$$
\begin{aligned}
\left\|\left(\lambda-u C_{\varphi}\right)^{2 n}: H X \rightarrow H X\right\| & \gtrsim\left\|\left(\lambda-u C_{\varphi}\right)^{2 n} f_{n}\right\|_{H X} \\
& \gtrsim\left\|\left(\lambda-u C_{\varphi}\right)^{2 n} f_{n}\right\|_{H^{1}} \\
& \gtrsim\left(1-\left|z_{n}\right|^{2}\right)\left|\left[\left(\lambda-u C_{\varphi}\right)^{2 n}\right] f_{n}\left(z_{n}\right)\right| .
\end{aligned}
$$

Now using the binomial expansion and formula (9) we obtain

$$
\begin{aligned}
{\left[\left(\lambda-u C_{\varphi}\right)^{2 n} f_{n}\right]\left(z_{n}\right) } & =\sum_{n=0}^{2 n}\left(\begin{array}{c}
2 n \\
k
\end{array}\right) \lambda^{2 n-k}\left[\left(-u C_{\varphi}\right)^{k} f_{n}\right]\left(z_{n}\right) \\
& =\sum_{k=0}^{2 n}\left(\begin{array}{c}
2 n \\
k
\end{array}\right) \lambda^{2 n-k}(-1)^{k} u_{(k)}\left(z_{n}\right) f_{n}\left(\varphi_{k}\left(z_{n}\right)\right) \\
& =\left(\begin{array}{c}
2 n \\
n
\end{array}\right)(-1)^{n} \lambda^{n} u_{(n)}\left(z_{n}\right) .
\end{aligned}
$$

Combining the above equality with (10) and using the substitution $w(z)=\frac{u(z)}{\varphi^{\prime}(z)}$ (note that $w_{(n)}(z)=u_{(n)}(z)\left(\varphi_{n}^{\prime}(z)\right)^{-1}$ by the proof of [11, Theorem 4.3]) we get

$$
\begin{aligned}
\left\|\left(\lambda-u C_{\varphi}\right)^{2 n}: H X \rightarrow H X\right\| & \gtrsim\left(\begin{array}{c}
2 n \\
n
\end{array}\right)|\lambda|^{n}\left|u_{(n)}\left(z_{n}\right)\right|\left(1-\left|z_{n}\right|^{2}\right) \\
& =\left(\begin{array}{c}
2 n \\
n
\end{array}\right)|\lambda|^{n}\left|u_{(n)}\left(z_{n}\right)\right|\left(\frac{1-\left|z_{n}\right|^{2}}{1-\left|\varphi_{n}\left(z_{n}\right)\right|^{2}}\right)\left(1-\left|\varphi_{n}\left(z_{n}\right)\right|^{2}\right) \\
& =\left(\begin{array}{c}
2 n \\
n
\end{array}\right)|\lambda|^{n}\left|\frac{u_{(n)}\left(z_{n}\right)}{\varphi_{n}^{\prime}\left(z_{n}\right)}\right|\left(1-\left|\varphi_{2 n}(0)\right|^{2}\right) \\
& \geqslant\left(\begin{array}{c}
2 n \\
n
\end{array}\right)|\lambda|^{n}\left|w_{(n)}\left(z_{n}\right)\right|\left(1-\left|\varphi_{2 n}(0)\right|\right) .
\end{aligned}
$$

In the proof of [11, Theorem 4.3] it was shown that

$$
\lim _{n \rightarrow \infty}\left|w_{(n)}\left(z_{n}\right)\right|^{\frac{1}{2 n}}=|u(a)|^{\frac{1}{2}} .
$$

Since

$$
\lim _{n \rightarrow \infty}\left(\begin{array}{c}
2 n \\
n
\end{array}\right)^{\frac{1}{2 n}}=2,
$$


we conclude that

$$
\begin{aligned}
r_{H X}\left(\lambda-u C_{\varphi}\right) & =\lim _{n \rightarrow \infty}\left\|\left(\lambda-u C_{\varphi}\right)^{2 n}: H X \rightarrow H X\right\|^{\frac{1}{2 n}} \\
& \geqslant \lim _{n \rightarrow \infty}\left(\begin{array}{c}
2 n \\
n
\end{array}\right)^{\frac{1}{2 n}}|\lambda|^{\frac{1}{2}}\left|w_{(n)}\left(z_{n}\right)\right|^{\frac{1}{2 n}}\left(1-\left|\varphi_{2 n}(0)\right|\right)^{\frac{1}{2 n}} \\
& =2|\lambda|^{\frac{1}{2}}|u(a)|^{\frac{1}{2}}=2|u(a)|=2 r_{H X}\left(u C_{\varphi}\right) .
\end{aligned}
$$

This proves that $r_{H X}\left(\lambda-u C_{\varphi}\right) \geqslant 2 r_{H X}\left(u C_{\varphi}\right)$, and hence by the Spectral Mapping Theorem $-\lambda \in \sigma_{H X}\left(u C_{\varphi}\right)$ showing that

$$
\{\lambda \in \mathbb{C}:|\lambda|=|u(a)|\} \subset \sigma_{H X}\left(u C_{\varphi}\right) .
$$

\subsection{The elliptic case}

The approach with invertible weighted composition operators induced by an elliptic automorphism is standard. We include the result and a sketch of the proof for the sake of completeness.

Theorem 4 Suppose that $u \in A(\mathbb{D})$ and $\varphi$ is an elliptic automorphism with the unique fixed point $a \in \mathbb{D}$.

(i) If there is a positive integer $j$ such that $\varphi_{j}(z)=z$ for all $z \in \mathbb{D}$, then letting $m$ be the smallest such integer, we have

$$
\sigma_{H X}\left(u C_{\varphi}\right)=\overline{\left\{\lambda \in \mathbb{C}: u_{(m)}(z)=\lambda^{m} \text { for some } z \in \mathbb{D}\right\}} .
$$

(ii) If $\varphi_{n} \neq \mathrm{Id}$ for every $n \in \mathbb{N}$ and if $u C_{\varphi}: H X \rightarrow H X$ is invertible, then

$$
\sigma_{H X}\left(u C_{\varphi}\right)=\{\lambda \in \mathbb{C}:|\lambda|=|u(a)|\} .
$$

Proof The proof of (i) is an easy modification of [11, Theorem 4.11].

To prove (ii) we follow the proof of [11, Theorem 4.14]. Namely, using the same methods we prove an analogue of [11, Lemma 4.13] which states that if $v \in A(\mathbb{D}), \psi(z)=e^{2 \pi i \theta} z$ where $\theta$ is irrational and if $v C_{\psi}: H X \rightarrow H X$ is invertible then

$$
r\left(v C_{\psi}: H X \rightarrow H X\right)=|v(0)| .
$$

Recall that if $\varphi: \mathbb{D} \rightarrow \mathbb{D}$ is an elliptic automorphism with fixed point $a \in \mathbb{D}$ and $\varphi_{a}: \mathbb{D} \rightarrow \mathbb{D}$ is an automorphism defined by $\varphi_{a}(z):=(a-z) /(1-\bar{a} z)$ then $\psi:=\varphi_{a} \circ \varphi \circ \varphi_{a}$ has zero as the fixed point and $\psi(z)=\varphi^{\prime}(a) z$ with $\left|\varphi^{\prime}(a)\right|=1$. Further if we put $v:=u \circ \varphi_{a}$ then $u C_{\varphi}$ and $v C_{\psi}$ are similar and we have $\sigma_{H X}\left(u C_{\varphi}\right)=\sigma_{H X}\left(v C_{\psi}\right)$. From the above we get

$$
r\left(u C_{\varphi}\right)=r\left(v C_{\psi}\right)=|v(0)|=\mid u\left(\left(\varphi_{a}(0)\right)|=| u(a) \mid .\right.
$$

We note that $\varphi^{-1}$ is an elliptic automorphism with fixed point $a \in \mathbb{D}$ such that $\varphi_{-n} \neq$ Id for $n \in \mathbb{N}$. Since $\left(u C_{\varphi}\right)^{-1}=\frac{1}{u \circ \varphi^{-1} e} C_{\varphi^{-1}}$, then $r\left(\left(u C_{\varphi}\right)^{-1}\right)=|u(a)|^{-1}$ and we easily obtain that $\sigma_{H X}\left(u C_{\varphi}\right) \subset\{\lambda \in \mathbb{C}:|\lambda|=|u(a)|\}$.

To show that $\{\lambda \in \mathbb{C}:|\lambda|=|u(a)|\} \subset \sigma_{H X}\left(u C_{\varphi}\right)$ notice that $e_{n}(z)=z^{n} \in H X$ for every $n \in \mathbb{N}_{0}$. Using a similar argument as in the proof of [13, Lemma 2.3] we obtain that $\sigma_{H X}\left(v C_{\psi}\right)$ contains $\left\{v(0) e^{2 \pi i n \theta}: n \in \mathbb{N}_{0}\right\}$ which is dense in $\{\lambda:|\lambda|=|u(a)|\}$. Thus

$$
\{\lambda:|\lambda|=|u(a)|\}=\{\lambda:|\lambda|=|v(0)|\} \subset \sigma_{H X}\left(v C_{\psi}\right)=\sigma_{H X}\left(u C_{\varphi}\right)
$$

and this completes the proof. 


\section{Spectra of weighted composition operators on Hardy-Lorentz spaces}

In this paragraph we consider a very general approach based on interpolation theory restricted to the special case of standard Hardy-Lorentz spaces. In particular we show that the spectrum of an operator on the Hardy-Lorentz space $H^{p, q}$ does not depend on the parameter $q$ and hence the spectra of weighted composition operators on Hardy-Lorentz $H^{p, q}$ and Hardy $H^{p}$ spaces are the same. This holds also for the general case when a weighted composition operator is not necessarily invertible.

Recall that for $p \in(1, \infty), q \in[1, \infty]$, the Hardy-Lorentz space $H^{p, q}$ is given by $H^{p, q}=$ $H L^{p, q}$.

Theorem 5 Let $\varphi$ be a holomorphic self-map of $\mathbb{D}, u \in A(\mathbb{D})$, and $p, q \in(1, \infty)$. Then

$$
\sigma_{H^{p, q}}\left(u C_{\varphi}\right)=\sigma_{H^{p}}\left(u C_{\varphi}\right) .
$$

Proof Notice that by [19, Proposition 1.4] and Theorem 1 the operator $u C_{\varphi}$ is bounded on $H^{p, q}$.

We apply the interpolation theorem on the stability of invertible operators on real interpolation spaces (see [1,2]), which states that if $q \in[1, \infty]$ and $T:\left(E_{0}, E_{1}\right) \rightarrow\left(F_{0}, F_{1}\right)$ is an operator between Banach couples and

$$
T:\left(E_{0}, E_{1}\right)_{\theta_{0}, q} \rightarrow\left(F_{0}, F_{1}\right)_{\theta_{0}, q}
$$

is invertible for some $\theta_{0} \in(0,1)$ then there exists $\varepsilon>0$ such that $\left(\theta_{0}-\varepsilon, \theta_{0}+\varepsilon\right) \subset(0,1)$ and

$$
T:\left(E_{0}, E_{1}\right)_{\theta, q} \rightarrow\left(F_{0}, F_{1}\right)_{\theta, q}
$$

is invertible for every $\theta \in\left(\theta_{0}-\varepsilon, \theta_{0}+\varepsilon\right)$.

To apply this result we recall the well-known formula

$$
\left(H^{1}, H^{\infty}\right)_{\theta, q}=H^{p, q}
$$

holding for all $\theta \in(0,1), q \in[1, \infty]$ with $1 / p=1-\theta$.

Fix $\lambda \notin \sigma_{H^{p, q}}\left(u C_{\varphi}\right)$, where $p<\infty, q \in[1, \infty]$. This means that the operator $T:=$ $\lambda I-u C_{\varphi}$ is invertible on $H^{p, q}$. Now taking $\theta_{0}:=1 / p^{\prime}:=1-1 / p$, by the above formula we conclude that

$$
T:\left(H^{1}, H^{\infty}\right)_{\theta_{0}, q} \rightarrow\left(H^{1}, H^{\infty}\right)_{\theta_{0}, q} .
$$

Since $T:\left(H^{1}, H^{\infty}\right) \rightarrow\left(H^{1}, H^{\infty}\right)$ is invertible it follows immediately from the mentioned stability result that there exist $p_{0}, p_{1} \in(1, \infty)$ such that $p \in\left(p_{0}, p_{1}\right)$ and

$$
T:\left(H^{1}, H^{\infty}\right)_{1 / p_{j}^{\prime}, q} \rightarrow\left(H^{1}, H^{\infty}\right)_{1 / p_{j}^{\prime}, q}
$$

is invertible for both $j=0,1$ or equivalently by (11)

$$
T: H^{p_{j}, q} \rightarrow H^{p_{j}, q}
$$

is invertible for $j=0,1$. Since $\left(H^{p_{0}, q}, H^{p_{1}, q}\right)$ is an ordered Banach couple, the inverses of $T$ coincide on $H^{p_{0}, q} \cap H^{p_{1}, q}=H^{p_{1}, q}$. This implies by interpolation that

$$
T:\left(H^{p_{0}, q}, H^{p_{1}, q}\right)_{s, r} \rightarrow\left(H^{p_{0}, q}, H^{p_{1}, q}\right)_{s, r}
$$

is invertible for all $s \in(0,1), r \in[1, \infty)$. 
Next we apply a formula which follows from a general interpolation result from [20]: For all $p_{0}, p_{1} \in(1, \infty), p_{0} \neq p_{1}, q_{0}, q_{1}, r \in[1, \infty], s \in(0,1)$,

$$
\left(H^{p_{0}, q_{0}}, H^{p_{1}, q_{1}}\right)_{s, r}=H^{p_{s}, r},
$$

where $1 / p_{s}=(1-s) / p_{0}+s / p_{1}$. Taking $s \in(0,1)$ such that $1 / p=(1-s) / p_{0}+s / p_{1}$ and $r=p$ we conclude that

$$
T: H^{p} \rightarrow H^{p}
$$

is invertible, i.e. $\lambda \notin \sigma_{H^{p}}\left(u C_{\varphi}\right)$. We have just proved that

$$
\sigma_{H^{p}}\left(u C_{\varphi}\right) \subset \sigma_{H^{p, q}}\left(u C_{\varphi}\right) .
$$

The reverse inclusion is obtained in a similar way and so

$$
\sigma_{H^{p}}\left(u C_{\varphi}\right)=\sigma_{H^{p, q}}\left(u C_{\varphi}\right) .
$$

From [11, Corollary 5.1] we obtain a description of the spectra of invertible weighted composition operators on Hardy-Lorentz spaces.

Corollary 1 Let $u C_{\varphi}$ be an invertible operator on Hardy-Lorentz space $H^{p, q}, p, q \in(1, \infty)$ and let $u \in A(\mathbb{D})$. Then

(i) if $\varphi$ is elliptic with fixed point $a \in \mathbb{D}$ then

(a) if there is a positive integer $j$ such that $\varphi_{j}(z)=z$ for all $z \in \mathbb{D}$, then letting $m$ be the smallest such integer, we have

$$
\sigma_{H^{p, q}}\left(u C_{\varphi}\right)=\overline{\left\{\lambda \in \mathbb{C}: u_{(m)}(z)=\lambda^{m} \text { for some } z \in \mathbb{D}\right\}},
$$

(b) if $\varphi_{n} \neq \operatorname{Id}$ for each $n \in \mathbb{N}$ and if $u C_{\varphi}: H X \rightarrow H X$ is invertible, then

$$
\sigma_{H^{p, q}}\left(u C_{\varphi}\right)=\{\lambda \in \mathbb{C}:|\lambda|=|u(a)|\} .
$$

(ii) if $\varphi$ is parabolic with fixed point $a \in \partial \mathbb{D}$, then

$$
\sigma_{H^{p, q}}\left(u C_{\varphi}\right)=\{\lambda \in \mathbb{C}:|\lambda|=|u(a)|\},
$$

(iii) if $\varphi$ is hyperbolic with attractive point $a \in \partial \mathbb{D}$ and repulsive point $b \in \partial \mathbb{D}$, then

$$
\begin{aligned}
& \sigma_{H^{p, q}}\left(u C_{\varphi}\right) \\
& =\left\{\lambda \in \mathbb{C}: \min \left\{\frac{|u(a)|}{\varphi^{\prime}(a)^{1 / p}}, \frac{|u(b)|}{\varphi^{\prime}(b)^{1 / p}}\right\} \leqslant|\lambda| \leqslant \max \left\{\frac{|u(a)|}{\varphi^{\prime}(a)^{1 / p}}, \frac{|u(b)|}{\varphi^{\prime}(b)^{1 / p}}\right\}\right\} .
\end{aligned}
$$

Let us also note that Theorem 5 can be applied to not necessarily invertible weighted composition operators. The corollary below follows from [12, Theorem 6] (cf. also [8]).

Corollary 2 Assume that $u, \varphi \in H(\mathbb{D}), \varphi(\mathbb{D}) \subset \mathbb{D}$, and a is a Denjoy-Wolff point with $\varphi_{n} \rightarrow$ a uniformly in $\mathbb{D}$ as $n \rightarrow \infty$ and $\varphi^{\prime}(a)<1$. If $u$ is bounded in $\mathbb{D}$ and continuous at $a$, then

$$
\sigma_{H^{p, q}}\left(u C_{\varphi}\right)=\left\{\lambda \in \mathbb{C}:|\lambda| \leqslant \frac{u(a) \mid}{\left|\varphi^{\prime}(a)\right|^{1 / p}}\right\} .
$$


Acknowledgements P. Mleczko's research was partially supported by the Foundation for Polish Science (FNP) and by the National Science Centre, Poland (Project no. 2015/17/B/ST1/00064). M. Rzeczkowski was partially supported by the Foundation for Polish Science (FNP).

Open Access This article is distributed under the terms of the Creative Commons Attribution 4.0 International License (http://creativecommons.org/licenses/by/4.0/), which permits unrestricted use, distribution, and reproduction in any medium, provided you give appropriate credit to the original author(s) and the source, provide a link to the Creative Commons license, and indicate if changes were made.

\section{References}

1. Asekritova, I., Kruglyak, N.: Necessary and sufficient conditions for invertibility of operators in spaces of real interpolation. J. Funct. Anal. 264(1), 207-245 (2013)

2. Asekritova, I., Kruglyak, N., Mastyło, M.: Interpolation of Fredholm operators. Adv. Math. 295, 421-496 (2016)

3. Bergh, J., Löfström, J.: Interpolation Spaces. A Series of Comprehensive Studies in Mathematics, vol. 223. Springer, Berlin (1976)

4. Bourdon, P.: Invertible weighted composition operators. Proc. Am. Math. Soc. 142, 289-299 (2013)

5. Chalendar, I., Gallardo, E., Partington, J.R.: Weighted composition operators on the Dirichlet space: boundedness and spectral properties. Math. Ann. 363(3-4), 1265-1279 (2015)

6. Cowen, C., MacCluer, B.: Composition Operators on Spaces of Analytic Functions. CRC Press, Boca Raton (1995)

7. Cowen, C.: Composition operators on $H^{2}$. J. Oper. Theory 9, 77-106 (1983)

8. Cowen, C., Ko, E., Thompson, D., Tian, F.: Spectra of some weighted composition operators on $H^{2}$. Acta Sci. Math. (Szeged) 82(1-2), 221-234 (2016)

9. Eklund, T., Lindström, M., Mleczko, P.: Spectral properties of weighted composition operators on the Bloch and Dirichlet spaces. Stud. Math. 232, 95-112 (2016)

10. Grafakos, L.: Classical Fourier Analysis, III edn. Graduate Texts in Mathematics, vol. 249. Springer, New York (2014)

11. Hyvärinen, O., Lindström, M., Nieminen, I., Saukko, E.: Spectra of weighted composition operators with automorphic symbols. J. Funct. Anal. 265, 1749-1777 (2013)

12. Hyvärinen, O., Nieminen, I.: Essential spectra of weighted composition operators with hyperbolic symbols. Concr. Oper. 2, 110-119 (2015)

13. Kamowitz, H.: Compact operators of the form $u C_{\varphi}$. Pac. J. Math. 80, 205-211 (1979)

14. Krein, S.G., Petunin, Yu.I., Semenov, E.M.: Interpolation of Linear Operators. Translations of Mathematical Monographs, vol. 54. American Mathematical Society, Providence (1982)

15. Lefèvre, P., Li, D., Queffélec, H., Rodríguez-Piazza, L.: Composition operators on Hardy-Orlicz spaces. Mem. Am. Math. Soc. 207(974), vi+74 (2010)

16. Lengfield, M.: Duals and envelopes of some Hardy-Lorentz spaces. Proc. Am. Math. Soc. 133(5), 14011409 (2005)

17. Lu, Q., Cao, G.F., Liu, L.F.: Hardy-Orlicz spaces and their multiplication operators. Acta Math. Sin. 21(3), 593-598 (2005)

18. Mastyło, M., Mleczko, P.: Norm estimates for matrix operators between Banach spaces. Linear Algebra Appl. 438(3), 986-1001 (2013)

19. Mastyło, M., Rodríguez-Piazza, L.: Carleson measures and embeddings of abstract Hardy spaces into function lattices. J. Funct. Anal. 268, 902-928 (2015)

20. Xu, Q.: Notes on interpolation of Hardy spaces. Ann. Inst. Fourier 42, 875-889 (1992) 